\title{
Tailored Molecular Imaging of Pheochromocytoma and Paraganglioma: Which Tracer and When
}

\author{
Giuseppe Danilo Di Stasio ${ }^{a}$ Vincenzo Cuccurullo ${ }^{b}$ Giuseppe Lucio Cascini ${ }^{c}$ \\ Chiara Maria Grana ${ }^{d}$ \\ ${ }^{a}$ Nuclear Medicine Service, Check-Up Polydiagnostic Center, Salerno, Italy; ${ }^{\text {b}}$ Nuclear Medicine Unit, Department of \\ Precision Medicine, Università della Campania "Luigi Vanvitelli", Napoli, Italy; 'Nuclear Medicine Unit, Department of

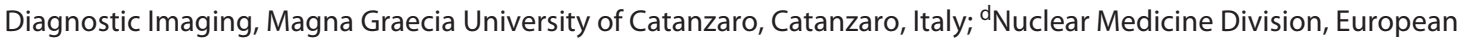 \\ Institute of Oncology, IRCCS, Milan, Italy
}

\section{Keywords}

Pheochromocytoma · Paraganglioma - Diagnostic imaging · Nuclear medicine $\cdot$ Theragnostics

\section{Abstract}

Pheochromocytoma (PCC) and paraganglioma (PGL) are rare neoplasms that fall within the category of neuroendocrine tumors. In the last decade, their diagnostic algorithm has been modified to include the evaluation of molecular pathways, genotype, and biochemical phenotype, in order to correctly interpret anatomical and functional imaging results and tailor the best therapeutic choices to patients. More specifically, the identification of germline mutations has led to a three-way cluster classification: pseudo-hypoxic cluster, cluster of kinase receptor signaling and protein translation pathways, and cluster of Wnt-altered pathway. In this context, functional imaging gained a crucial role in the management of these patients in agreement with the evergrowing concept of personalized medicine. In this paper, we provide an overview of three specific molecular pathways targeted by positron-emitting tracers to image PCCs and PGLs: catecholamine metabolism, somatostatin receptors, and glucose uptake. Finally, we recommend different flow charts for use in the selection of tracers for specific clinical scenarios, based on sporadic/inherited tumor and known/ unknown mutation status.

(c) 2022 S. Karger AG, Base

\section{Introduction}

Pheochromocytoma (PCC) is a rare neoplasm belonging to the category of neuroendocrine tumors (NETs). It has an incidence of $2-8$ cases per $1,000,000$ per year, originates from the neural crest, and arises from chromaffin cells of adrenal glands medulla (90\%). However, it can also be located in extra-adrenal chromaffin tissue (10\%), in particular deriving from sympathetic nervous system ganglia, in which case it is referred to as paraganglioma (PGL), named according to the primary anatomical site of origin [1]. Their main feature is the synthesis and secretion of high amounts of catecholamines, mostly norepinephrine (NE), followed by epinephrine (E) and dopamine (DA), the latter being primarily associated with malignancy [2]. From an epidemiologic point of view, its sporadic form mostly occurs in middle-age adults (approximately 50 years), although it presents earlier in he- 
reditary cases, which are mainly related to succinate dehydrogenase subunits (SDHx), multiple endocrine neoplasia (MEN2), von Hippel-Lindau disease (VHL), or neurofibromatosis type 1 (NF1) [3]. In this sense, the importance of genetic mutations in PCCs and PGLs (PPGLs) has evolved over recent decades, bringing the previously considered $10 \%$ of genetic etiology (i.e., the sum of germline and somatic mutations) to almost $70 \%$ of mutations regarding more than 20 genes associated with these tumors and up to $80 \%$ in some age-groups ( $\leq 18$ years) [4]. Therefore, mainly for patients with high-risk syndromes and germline mutations or with biochemical evidence of disease, diagnostic imaging plays a fundamental role not only for diagnosis and staging purposes but also in restaging, selection for targeted molecular radiotherapy, and follow-up [5]. In particular, diagnostic imaging can be performed to localize primary lesions and/or to assess for any metastatic disease with computed tomography (CT) or magnetic resonance imaging (MRI) alone, such as in anatomical imaging of the abdomen and pelvis, or combined with whole-body positron emission tomography (PET) as in PET/CT or PET/MRI, with functional imaging being the best next step if morphology is negative, and clinical suspicion is still high [6].

Given this extremely variable picture, it is clear how the diagnostic algorithm has to focus primarily on patient history and this clinical evaluation. However, as recent literature data shows, it is crucial to implement a thorough evaluation of molecular pathways, genotype, and biochemical phenotype, in order to correctly interpret anatomical and functional imaging results and tailor the best therapeutic choices to patients, whether these choices be surgical, pharmacological, or theragnostic [7]. The aim of this paper was to provide an overview of tailored nuclear medicine imaging possibilities based on metabolic pathways targeted by positron-emitting tracers and to help physicians in everyday clinical practice select tracers for specific clinical scenarios, based on sporadic/inherited tumor and known/unknown mutation status.

\section{Molecular Pathways, Genetics, and Biochemical Phenotype}

As 2019 EANM/SNMMI Practice Guidelines for radionuclide imaging of PPGLs report [8], the presence of multiple PCCs or the combination of PCC with a synchronous or metachronous extra-adrenal PGL is associated with germline/somatic mutations in more than $70 \%$ of cases. It is therefore crucial to recognize the most im- portant correlations between genes and specific anatomical predispositions [8].

In addition to VHL and NF1 genes [9], other germline mutations have been identified in SDHx, rearranged during transfection (RET), hypoxia-inducible factor-2 alpha (HIF-2 $\alpha$ ), Myc-associated protein X (MAX), and several more, which have led to a cluster classification of PPGLs based on the molecular pathways involved:

1. Pseudo-hypoxic cluster (mutations in SDHx, FH, MDH2, VHL, and HIF-2 $\alpha$ )

2. Cluster of kinase receptor signaling and protein translation pathways (mutations in RET, NF1, MAX, and HRAS)

3. Cluster of Wnt-altered pathway that includes newly recognized somatic mutations in cold shock domain containing E1 and cause aggressive forms of sporadic PCC [10].

Moreover, inactivation of ATRX or activation of TERT (genes related to telomere maintenance) or mutations in SETD2 (chromatin maintenance) also appear to impact the clinical evolution of the disease [11]. The first cluster is characterized by an overactivation of hypoxia signaling that could depend on two subgroups of mutations (1A and $1 \mathrm{~B}): 1 \mathrm{~A}$ is related to a general state of hypoxia and derives from loss-of-function mutations in SDHx and $\mathrm{FH}$ genes, thus leading to the accumulation of Krebs cycle precursors such as fumarate and succinate, which act as oncometabolites upregulating glucose metabolism, proliferation, angiogenesis, and erythropoiesis [12]. 1B, on the other hand, is related to mutations in genes of hypoxia-signaling pathway and include VHL and HIF-2 $\alpha$. Lossof-function mutations in VHL lead to HIF-2 $\alpha$ accumulation, thus promoting angiogenesis, tumor spreading, and metastasis [13]. The second cluster is mainly characterized by RET and NF1 mutations, although HRAS, transmembrane protein 127, MAX, and fibroblast growth factor receptor 1 mutations have been reported [14]. RET encodes a tyrosine kinase receptor and is physiologically involved in RAS/MAPK and PI3K/AKT transduction pathways of proliferation, survival, and angiogenesis; therefore, gain-of-function mutations in RET lead to constitutive activation and consequent deregulation of such pathways determining increased cell survival. NF1 instead is a tumor suppressor gene that physiologically inhibits RAS and mTOR kinase pathways; hence, a lossof-function mutation at this level leads to enhanced cell proliferation and cancer development (Table 1).

With respect to PCC, the most common mutations reported are related to RET proto-oncogene, VHL, SDHx, NF1, and MAX, whereas PGLs with an underlying muta- 
tion in subunit B of SDH (SDHB) are associated with a higher risk of aggressive behavior and development of metastases [14]. Moreover, the involvement of specific molecular pathways could imply the presence of PPGLs as part of syndromic presentation or even suggest bilateral tumors, the presence of metastatic disease, or pediatric onset. In this sense, most common syndromes are VHL, MEN2, NF1, and those associated with HIF-2 $\alpha$ mutations: bilateral PCC is usually related to MEN2 or VHL disease, metastatic presentation is associated mainly with SDHB and cluster 3-related genes such as "mastermindlike" transcriptional coactivator 3 , whereas the pediatric onset of PPGLs has the highest prevalence of genetic mutations (up to $80 \%$ ) which are most frequently found on SDHB, SDHD, and VHL genes (cluster 1 genes) [15]. Cluster-2-related tumors instead have a low metastatic risk being almost exclusively located adrenally [16].

PPGLs can also be classified according to their biochemical phenotypes which relate to the hypersecretion of different combinations of catecholamines: only NE, both NE and DA, both E and NE, or solely E or DA (rare). In this sense, The pattern of PPGL catecholamine secretion could be helpful in recognizing their genetic background (Table 1) [17]. Cluster 1 gene mutations are more often associated with a noradrenergic and/or dopaminergic secretion with low catecholamine content constantly released due to HIF- $2 \alpha$ activity that in case of hypoxia, favors the production of NE in the catecholamine production pathway. This is due to the activation of enzymes such as tyrosine hydroxylase, DA ß-hydroxylase, and dopa-decarboxylase and the contemporary reduction of expression of phenylethanolamine N-methyltransferase, which converts NE to E [11]. This is the case in VHL mutations, in which, tumors present only NE hypersecretion, independently of whether the location is adrenal or extra-adrenal, or in PPGLs from patients with SDHBand SDHD-mutated genes that are characterized by overproduction of DA, either with or without NE, although SDHB-related extra-adrenal abdominal PPGLs could also be biochemically silent [14]. Gene mutations in cluster 2 include MEN2 and NF1 patients whose PPGLs tend to be intra-adrenal and rarely malignant. In this group, deriving tumors are related almost exclusively to an adrenergic phenotype with high catecholamine content and episodic secretion due to RET or NF1 mutations that determine an increase in phenylethanolamine N-methyltransferase, which ultimately leads plasma metanephrines values to exceed more than 5\% the combined increases of both metabolites [15]. Cluster 3-related tumors finally, showed the highest chromogranin A overexpres- 
sion, although the catecholamine phenotype is mostly unknown. Therefore, taking also into consideration the elevated costs of genetic testing, biochemical phenotypes could furnish useful information when selecting which genes to analyze as a first-line approach $[18,19]$.

\section{Clinical Presentation and Diagnosis}

The clinical presentation of PPGLs is highly variable. The "classic" triad is characterized by episodic "pounding" headache, palpitations/tachycardia, and diaphoresis. However, the full clinical picture includes other symptoms (tremor, anxiety, chest or abdominal pain, nausea/ vomiting, visual blurring, and weight loss) and signs (orthostatic hypotension and papilledema) [20]. However, the most relevant clinical finding is paroxysmal or persistent hypertension due to overproduction and secretion of catecholamines and their precursors [21]. Paroxysmal crises can be triggered by emotive stimuli or anxiety or physical activities that shrink the tumor mass (such as physical exercise, change of position, mass palpation, and defecation), causing a sudden release of catecholamines. It is crucial to follow a precise diagnostic and therapeutic algorithm because cardiovascular morbidity and mortality arising from catecholamine hypersecretion are elevated in untreated patients [22]. NE is metabolized to normetanephrine, and $\mathrm{E}$ is metabolized to metanephrine. Since this process takes place inside the tumor independently of catecholamine release, the North American Neuroendocrine Tumor Society (NANETS) recommends the measurement of plasma free metanephrines or urinary fractioned metanephrines as the first biochemical screening test for PCCs and PPGLs, rather than measuring parent catecholamines and vanillylmandelic acid. In fact, plasma metanephrine testing has the highest sensitivity (96\%) for detecting a PCC, but it has a lower specificity (85\%) [23]. In comparison, a 24-h urinary collection for catecholamines and metanephrines has a sensitivity of $87.5 \%$ and a specificity of $99.7 \%$ [23].

As stated in the 2019 EANM/SNMMI Practice Guidelines for radionuclide imaging of PPGLs, imaging studies should be performed for confirmation and localization of disease, when biochemical testing has proved clear evidence of PCC or PPGL [8]. However, in some situations such as head-and-neck PPGLs (HNPGLs), biochemical evidence may be absent, and patients could lack any symptoms, thus representing a significant diagnostic challenge with higher risk of advanced metastatic disease [24]. Visualization of these tumors is performed via CT or MRI, whereas scintigraphy and PET scanning could be employed when conventional imaging techniques fail to localize them [25]. In particular, abdominal CT has an 85-95\% accuracy for detecting adrenal masses with a spatial resolution of $1 \mathrm{~cm}$ or greater but is less accurate for lesions smaller than $1 \mathrm{~cm}$ [26]. While most PCCs have CT attenuation greater than 10 Hounsfield units $(\mathrm{HU})$, they rarely show sufficient intracellular fat to have an attenuation of less than $10 \mathrm{HU}$. In addition, most PCCs are hypervascular but have variable enhancement loss. Therefore, in patients in whom PCCs are strongly suspected, adrenal PCCs cannot be entirely excluded from the list of differential diagnoses if an attenuation value of less than $10 \mathrm{HU}$ and a washout greater than $60 \%$ on 15 -min delayed scanning are present [26]. Although it has been thought that the use of intravenous contrast poses a risk of inducing hypertensive crisis in patients with PCCs, it has to be noted that several studies concluded that this use of IV contrast is safe, even in patients not receiving alphaor $\beta$-blockers. Concerning MRI, this technique is preferred for PCC detection in children and in pregnant or lactating women as it does not require contrast and does not expose the patient to ionizing radiation, while still reporting a sensitivity of up to $100 \%$ in detecting adrenal PCCs. In approximately $70 \%$ of cases, PCCs appear hyperintense on T2-weighted images because of their highwater content [27]. Therefore, anatomical imaging could be sufficient for PPGL confirmation, especially if the metanephrine level is very high, and there is only adrenal gland involvement. However, since NE and subsequently normetanephrine could derive also from sympathetic nerves present anywhere in the body, if the level of this biochemical marker is found to be increased, functional imaging is required to confirm both the localization and the extent of disease [28].

\section{Nuclear Imaging}

In recent decades, the development of PET tracers and implementation of personalized medicine have led to an increased importance of functional imaging in the management of patients with PPGL since it provides wholebody in vivo characterization of tumors on a molecular level, thus mimicking ex vivo histological classification [29-31]. The 2019 EANM/SNMMI Practice Guidelines for radionuclide imaging of PPGLs report several clinical indications, including confirmation of PPGL diagnosis, initial staging, restaging, follow-up, and selection of patients for targeted molecular radiotherapy. In this sense, 
Table 2. Tracers for radionuclide imaging of PPGLs according to the metabolic pathway and imaging technique

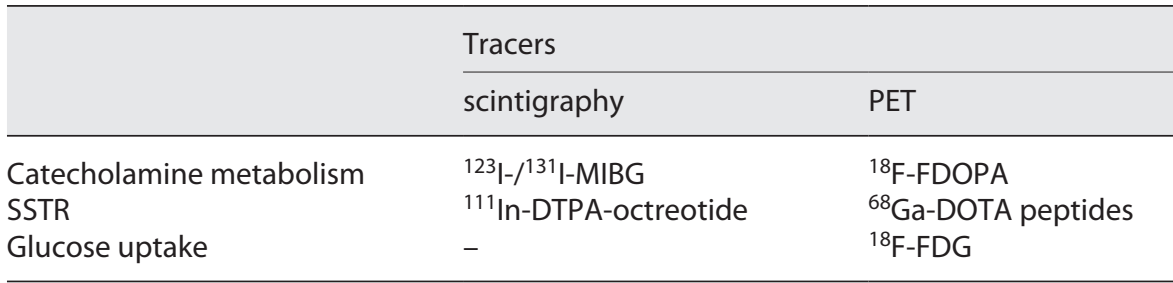

there are currently three main metabolic pathways involved in the pathogenesis of these tumors that are targeted by different positron-emitting radiopharmaceuticals: catecholamine metabolism, somatostatin receptor (SSTR), and glucose uptake imaging (Table 2).

\section{Catecholamine Metabolism Imaging}

Scintigraphy scanning with ${ }^{123}$ I-metaiodobenzylguanidine (MIBG) still represents the first choice of nuclear medicine imaging for PPGLs [32]. MIBG is a substrate for the NE transporter and concentrates within intracellular storage granules via vesicular monoamine transporter (VMAT) 1 and VMAT2 of cells of neuroectodermal origin, such as those of adrenal or extra-adrenal PPGLs. Moreover, since $1983,{ }^{123}$ I-MIBG has been used apart from its diagnostic utility, in the form of ${ }^{131} \mathrm{I}-\mathrm{MIBG}$, for therapeutic purposes in chromaffin tumors and NETs [33]. In cases of adrenal PCCs, ${ }^{123}$ I-MIBG scintigraphy shows high sensitivity and specificity values, of $90 \%$ and $95 \%$, respectively, which increase to approximately $100 \%$ when combined with catecholamine measurements [34]. Therefore, especially when using hybrid SPECT/CT cameras, which increase overall sensitivity and diagnostic potential of $\gamma$-emitting tracers, ${ }^{123}$ I-MIBG whole-body scintigraphy is particularly helpful in localization of primary lesions and definition of disease burden in terms of distant metastases [35]. This statement is particularly true in cases in which a PCC is confirmed biochemically, but CT or MRI scans do not show a tumor or in the detection of recurrences after surgical treatment [36]. Nonetheless, practical constraints such as long imaging times and lower sensitivity for extra-adrenal tumors (58\%) remain important limitations for ${ }^{123} \mathrm{I}-\mathrm{MIBG}$ imaging. More recently, catecholamine metabolism imaging shifted to PET/CT that granted faster imaging protocols and improved diagnostic accuracy in terms of both sensitivity and specificity thanks to the introduction of positron-emitting tracers such as ${ }^{18} \mathrm{~F}$-di-idrossiphenilalanine $\left({ }^{18} \mathrm{~F}\right.$-FDOPA) [37]. In consideration of its molecular pathway (shown in Fig. 1), although initially introduced in clinical practice to investigate the dopaminergic system of substantia nigra,
${ }^{18} \mathrm{~F}$-FDOPA has gained increasing importance in oncology due to increased expression of amino acid transporters and use of amino acids for protein synthesis and proliferation of cancer cells [38].

With respect to PPGLs, abundant data from the published literature demonstrate the diagnostic accuracy of ${ }^{18} \mathrm{~F}$-FDOPA PET/CT as an integrated functional and morphological imaging tool at any stage of the disease. In this context, the 2019 EANM/SNMMI Practice Guidelines for radionuclide imaging of PPGLs suggest for maximum tumor uptake and the acquisition of static images at 20 min after injection, although SUVmax still remains at $80 \%$ of the maximum value after $132 \mathrm{~min}$ [8]. Some authors use premedication with $200 \mathrm{mg}$ of carbidopa $1 \mathrm{~h}$ before the ${ }^{18} \mathrm{~F}$-FDOPA injection, which acts as an AADC inhibitor, in order to improve the bioavailability of the tracer and decrease physiological uptake by the pancreas; however, this is not recommended in the setting of PPGLs [39]. Fottner et al. [40] prospectively evaluated 30 patients with suspected PPGL in order to correlate functional imaging results with biochemical, molecular-genetic, and immunohistochemical findings. In all patients, they performed CT and/or MRI, ${ }^{123}$ I-MIBG scintigraphy and ${ }^{18} \mathrm{~F}$-FDOPA PET/CT and identified 64 lesions with both functional imaging modalities; however, ${ }^{18} \mathrm{~F}-\mathrm{FDO}$ $\mathrm{PA}$ PET/CT proved to be much more sensitive than ${ }^{123} \mathrm{I}-$ MIBG, identifying 62 lesions versus 34, respectively. In particular, comparable sensitivities were found for adrenal and abdominal lesions, whereas in thoracic/cervical lesions, ${ }^{123}$ I-MIBG sensitivity dropped to $15 \%$, compared to $100 \%$ for ${ }^{18} \mathrm{~F}$-FDOPA. Therefore, they determined overall sensitivity and specificity values of ${ }^{18} \mathrm{~F}$-FDOPA PET/CT 98\% and 100\%, respectively. Interestingly, with respect to molecular and immunohistochemical links to functional imaging, they demonstrated that VMAT differential expression is closely correlated with both ${ }^{123} \mathrm{I}-$ MIBG and ${ }^{18} \mathrm{~F}$-FDOPA. In particular, VMAT-1 expression was found to be essential for a positive ${ }^{123}$ I-MIBG scintigraphy since all VMAT-1-negative tumors on immunohistochemical analysis were also undetected on ${ }^{123}$ I-MIBG imaging. Conversely, ${ }^{18}$ F-FDOPA PET/CT 


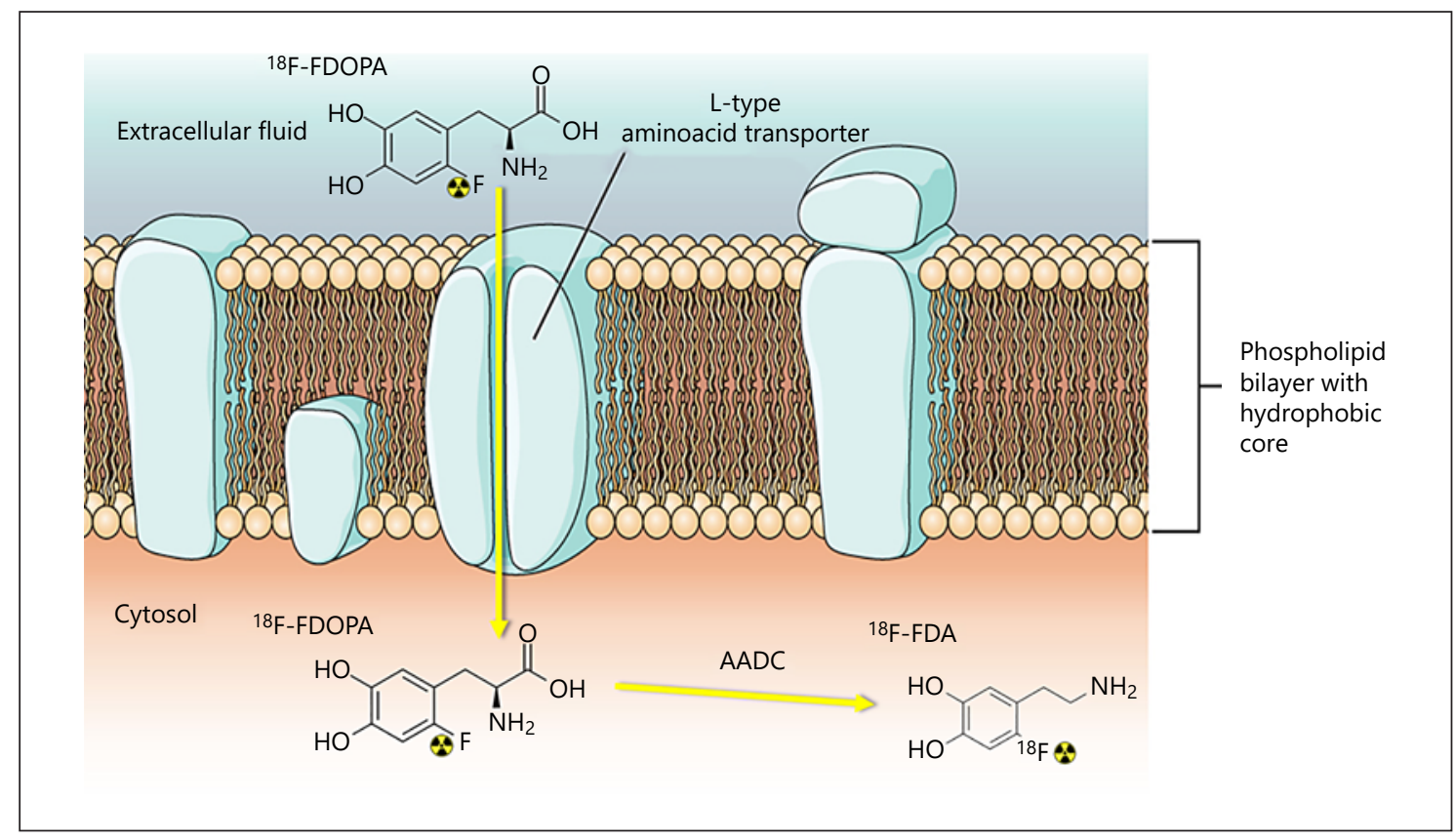

Fig. 1. Molecular pathway of ${ }^{18} \mathrm{~F}-\mathrm{FDOPA}$, amino acid PET tracer and precursor of L-DOPA. The L-type amino acid transporter is responsible for intracellular uptake, and aromatic amino acid decarboxylase (AADC) converts ${ }^{18} \mathrm{~F}$-FDOPA into ${ }^{18} \mathrm{~F}$-Dopamine $\left({ }^{18} \mathrm{~F}\right.$-FDA $)$.

was able to identify PPGLs that presented VMAT-2 alone, even in the absence of VMAT-1, since all ${ }^{123}$ I-MIBG-negative tumors were detected by ${ }^{18} \mathrm{~F}$-FDOPA PET/CT scans [40].

More recently, in a 2019 retrospective study of 45 patients with histologically confirmed PCCs, the investigators tried to determine SUVmax cutoff values for normal adrenal ${ }^{18} \mathrm{~F}$-FDOPA uptake using both intraindividual analysis (comparison with the contralateral adrenal gland) and interindividual analysis. All patients underwent ${ }^{18} \mathrm{~F}$-FDOPA PET/CT for localization purposes before adrenalectomy, whereas the control group consisted of 245 patients who underwent the same exam for other indications and without any genetic susceptibility for developing a PCC. Their results showed a median SUVmax in PCCs of 12 (range 2.6-50), while there was no significant difference in the control group between SUVmax of the left and right adrenal glands with a median SUVmax of 2.9 (range 1.1-6.6). ROC analysis was performed based on these results, and it identified the best value for SUVmax cutoff at 4.1 , with $93 \%$ sensitivity and $85 \%$ specificity (area under the curve $=0.951$ ), whereas in terms of intraindividual analysis, sensitivity of $93 \%$ and specificity 96\% were found for a difference in SUVmax between the affected gland and normal contralateral of 1.0 (area under the curve $=0.992)$. Therefore, they concluded that ${ }^{18} \mathrm{~F}$ FDOPA PET/CT has the highest diagnostic accuracy in the localization of PCCs when combining both parameters, namely absolute SUVmax and difference in SUVmax between the affected and normal adrenal gland [41].

Nonetheless, despite ${ }^{18}$ F-FDOPA, high diagnostic accuracy in detection of PPGLs and false negative results may occur, especially in abdominal tumors [42]. As a recent retrospective study by Gabriel et al. [42] demonstrated, these missed lesions on ${ }^{18} \mathrm{~F}$-FDOPA PET/CT are often associated with SDHx mutations, thus suggesting the possibility of specific genetic screening for SDHx mutations in patients with confirmed PPGLs but ${ }^{18} \mathrm{~F}$-FDOPA PET/ CT-negative. In detail, the investigators studied 116 patients with nonmetastatic PPGL who underwent genetic screening for SDHx and VHL mutations and ${ }^{18} \mathrm{~F}-\mathrm{FDOPA}$ PET/CT. Tracer uptake was considered pathologic if there was asymmetric adrenal uptake with a concordant enlarged gland, adrenal uptake more intense than that of the liver, or nonphysiological extra-adrenal focal uptake; moreover, lesions were classified into sympathetic and parasympathetic by location, according to embryologic development. When available, results were also compared with ${ }^{18} \mathrm{~F}$-fluorodeoxyglucose $\left({ }^{18} \mathrm{~F}\right.$-FDG) PET/CT reports (103 cases out of 116). Their data revealed that nearly $40 \%$ 
of patients harbored germline mutations, mostly associated with SDH subunits $\mathrm{B}, \mathrm{C}$, and $\mathrm{D}$, and that ${ }^{18} \mathrm{~F}$-FDOPA $\mathrm{PET} / \mathrm{CT}$ had patient and lesion-based sensitivities of 92.2\% and 91.8\%, respectively. Higher diagnostic values were found for the detection of parasympathetic PGLs rather than sympathetic PGLs, which proved to be also more at risk for a negative ${ }^{18} \mathrm{~F}-\mathrm{FDOPA} \mathrm{PET} / \mathrm{CT}$ scan in cases of concomitant SDHx mutations. Consequently, with respect for false negative cases, their analysis showed that among patients with adrenal and/or extra-adrenal abdominal PPGL, false-negative ${ }^{18} \mathrm{~F}$-FDOPA PET/CT scans only occurred in SDHx patients, and in all cases, missed lesions were detected by ${ }^{18} \mathrm{~F}-\mathrm{FDG}$ PET/CT. Therefore, they concluded that ${ }^{18} \mathrm{~F}-\mathrm{FDOPA} \mathrm{PET} / \mathrm{CT}$ presents high sensitivities in sympathetic nonmetastatic SDHB-related PPGL, whereas in cases of sympathetic metastatic SDHBrelated PGLs tumor, dedifferentiation could determine higher ${ }^{18}$ F-FDG and lower ${ }^{18}$ F-FDOPA uptake [42].

\section{SSTR Imaging}

SSTR imaging has always been considered the gold standard imaging technique for NETs [43]. Radiolabeled somatostatin analogs (SSAs) such as ${ }^{111}$ In-DTPA-octreotide $\left(\right.$ Octreoscan ${ }^{\circledR}$ ), specifically bind to SSTR subtypes (most commonly SSTR2 and SSTR5) expressed on the surface of neuroendocrine cells, allowing functional in vivo imaging of the tumor [44-46]. With respect to PPGL, they may overexpress SSTR; therefore, radiolabeled SSAs have been proposed in these subjects. Octreoscan ${ }^{\circledR}$ showed a greater sensitivity for the detection of metastatic PCC compared to benign tumors, even though with low overall sensitivity ( $30 \%)$, thus leaving it as a second line approach for PPGL patients [47]. However, thanks to the introduction of ${ }^{68} \mathrm{Gallium}$-labeled peptides, SSTR imaging received a major impulse to its diffusion not only for NETs imaging in general but also for PPGLs [48]. Once eluted, ${ }^{68} \mathrm{Ga}$ is linked to different SSAs through a chelating agent, more frequently DOTA, which acts as a universal bifunctional chelator able to form stable complexes with different radiotracers of the metal group like ${ }^{111} \mathrm{In},{ }^{68} \mathrm{Ga},{ }^{64} \mathrm{Cu},{ }^{90} \mathrm{Y}$, and ${ }^{177} \mathrm{Lu}$, with the last two radioisotopes used for radionuclide therapy [49]. In this group, three radiotracers are currently the most used in clinical practice: ${ }^{68} \mathrm{Ga}$-DOTA-NOC $\quad\left({ }^{68} \mathrm{Ga}\right.$-DOTA,1-Nal3-octreotide), ${ }^{68} \mathrm{Ga}$-DOTA-Tyr3-octreotide $\left({ }^{68} \mathrm{Ga}\right.$-DOTATOC), and ${ }^{68} \mathrm{Ga}$-DOTA-Tyr3-octreotate $\left({ }^{68} \mathrm{Ga}\right.$-DOTATATE) [50]. ${ }^{68} \mathrm{Ga}$-DOTA peptides have great in vivo stability, good pharmacokinetic properties, and high and specific receptor-mediated tumor uptake, about ten times higher compared to Octreoscan ${ }^{\circledR}$; in particular, ${ }^{68} \mathrm{Ga}$ -

Tailored Molecular Imaging of

Pheochromocytoma and Paraganglioma
DOTA-TATE has the highest affinity toward SSTR2, ${ }^{68} \mathrm{Ga}$-DOTA-TOC binds also with high affinity to SSTR5, whereas ${ }^{68} \mathrm{Ga}$-DOTA-NOC has the wider spectrum of affinity for SSTRs (SSTR2, SSTR3, and SSTR5) [51]. Even though current clinical studies reported similar performances, a full interchangeability is not suggested between them, and the choice of the same radiotracer during follow up of individual patients is indicated, depending on availability [52]. Unfortunately, despite the diagnostic accuracy of ${ }^{68} \mathrm{Ga}$-DOTA peptides, which proved to be excellent in localization of PPGLs mainly when metastatic or extra-adrenal, literature data are scarce and restricted to a small cohort of patients [53]. Maurice et al. [54] compared the sensitivity of ${ }^{68} \mathrm{Ga}$-DOTA-TATE PET/CT with ${ }^{123}$ I-MIBG scintigraphy in 15 patients with PPGLs, finding concordant results only in 8 out of 15 patients and concluding that the former should be considered as a first-line investigation in high-risk patients and in case of metastatic disease where genetic mutations associated with PPGL syndromes cannot be excluded. In this sense, Janssen et al. [55] assessed the clinical utility of ${ }^{68} \mathrm{Ga}-\mathrm{DO}$ TA-TATE PET/CT over anatomical imaging (CT/MRI) and other functional imaging modalities including ${ }^{18} \mathrm{~F}$ FDG and ${ }^{18} \mathrm{~F}$-FDOPA PET/CT in a prospective study of 20 patients with SDHx (SDHB and/or SDHD)-related and sporadic HNPGLs. Thirty-seven lesions were identified by ${ }^{18} \mathrm{~F}$-FDOPA PET/CT which was used as standard of reference and proved to be superior to ${ }^{18} \mathrm{~F}-\mathrm{FDG}$ PET/ CT. However, ${ }^{68} \mathrm{Ga}$-DOTA-TATE PET/CT was the most sensitive which identified significantly more lesions compared to all other imaging modalities performed [55]. In another study from the same group, the investigators focused on a cohort of 17 patients with SDHB-related metastatic PCC/PGL. Their results showed a lesion-based detection rate for ${ }^{68} \mathrm{Ga}$-DOTA-TATE PET/CT of $98.6 \%$, compared to $85.8 \%$ for ${ }^{18} \mathrm{~F}-\mathrm{FDG}$ and $61.4 \%$ for ${ }^{18} \mathrm{~F}-\mathrm{FDO}$ $\mathrm{PA}$, identifying 285 of 289 lesions and 33 additional lesions compared to any other functional imaging modality used in the study [56]. As a recent study pointed out, the superiority of ${ }^{68} \mathrm{Ga}$-DOTA-TATE over ${ }^{18} \mathrm{~F}$-FDOPA mainly in case of metastatic PPGLs and SDHx mutations could be explained by an overexpression of SSTR2A and SSTR3 in patients with SDH deficiency despite the high malignant potential and presumed dedifferentiation instead could determine reduction of large amino acid transporters [57]. Moreover, besides its diagnostic value, ${ }^{68} \mathrm{Ga}$-DOTA-TATE PET/CT could be used to determine which patients may benefit of peptide receptor radionuclide therapy, especially in metastatic disease or when surgery is not an option (shown in Fig. 2) [58]. The inves-

Neuroendocrinology 2022;112:927-940 


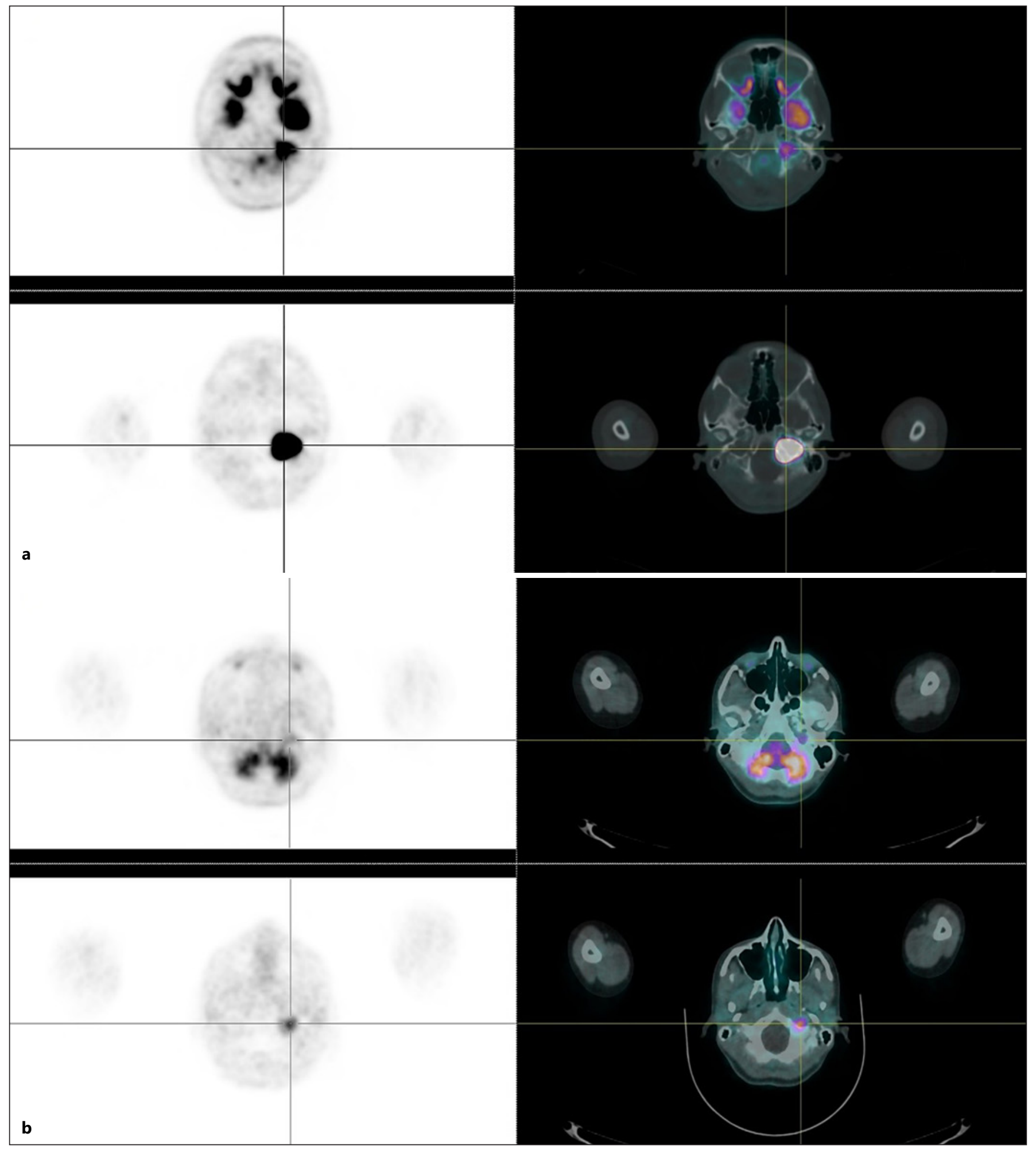

Fig. 2. Basal (a) and post-therapy (b) PET/CT scans with ${ }^{18} \mathrm{~F}-\mathrm{FDG}$ (up) and ${ }^{68} \mathrm{Ga}$-DOTATOC (down) of patient with HNPGL and SDHB mutation treated with five cycles of ${ }^{177} \mathrm{Lu}$-DOTATATE. The post-therapy scan shows partial response to treatment with residual moderate ${ }^{68} \mathrm{Ga}$-DOTATOC uptake and almost absent ${ }^{18} \mathrm{~F}-\mathrm{FDG}$ uptake. 
Table 3. Selection of tracers according to the tumor site

\begin{tabular}{|c|c|c|c|}
\hline${ }^{123}$ I-MIBG & Adrenal and abdominal & VMAT1+ & Not indicated if VMAT1- \\
\hline${ }^{18} \mathrm{~F}-\mathrm{FDOPA}$ & Total body & $V M A T+n o n-S D H x$ & $\begin{array}{l}\text { Indicated also if VMAT1- } \\
\text { Less indicated in cases of SDHx mutations }\end{array}$ \\
\hline${ }^{68} \mathrm{Ga}-\mathrm{DOTA}$ peptides & HNPGL/extra-adrenal & $S D H x$ & Best in metastatic PPGLs \\
\hline${ }^{18} \mathrm{~F}-\mathrm{FDG}$ & Total body & $S D H x$ & Best in metastatic PPGLs \\
\hline
\end{tabular}

Table 4. Selection of most sensitive PET/CT tracers according to molecular cluster

\begin{tabular}{lllll}
\hline & Cluster 1A (SDHx-related) & Cluster 1B (VHL-related) & Cluster 1B (HIF-2a-related) & Cluster 2 \\
\hline First choice & ${ }^{68}$ Ga-DOTA-SSA & ${ }^{18}$ F-FDOPA & ${ }^{18}$ F-FDOPA & Cluster 3 \\
Second choice & ${ }^{18}$ F-FDG & ${ }^{68}$ Ga-DOTA-SSA & ${ }^{18}$ F-FDG & ${ }^{68}$ Ga-DOPA \\
\hline
\end{tabular}

tigators therefore concluded that such promising results linked to the increasing availability of DOTA analogs should lead them to become the preferred functional imaging tracers in this specific scenario, even in pediatric patients [59]. The 2019 EANM/SNMMI Practice Guidelines for radionuclide imaging of PPGLs report seven studies that compare the performance of ${ }^{68} \mathrm{Ga}$-DOTASSA PET/CT and ${ }^{18}$ F-FDOPA PET/CT but distinguish between the diagnostic accuracy of the tracers according to the clinical setting of PCC rather than PGL. In the former scenario, ${ }^{18} \mathrm{~F}$-FDOPA PET/CT showed better patient-based and lesion-based detection rates than ${ }^{68} \mathrm{Ga}$ DOTA-SSA PET/CT, but in published studies involving patients with extra-adrenal PPGLs, detection rates reversed, with $98 \%$ for ${ }^{68} \mathrm{Ga}$-DOTA-SSA and $95 \%$ for ${ }^{18} \mathrm{~F}$ FDOPA; analogously, with respect to SDHx-associated PPGL lesions, gallium-based peptides showed higher sensitivity than ${ }^{18}$ F-FDG PET/CT [8].

\section{Glucose Uptake Imaging}

Due to its nonspecific accumulation, ${ }^{18} \mathrm{~F}-\mathrm{FDG}$ is not considered as the tracer of choice for PPGLs imaging, although several studies reported superior sensitivity over CT/MRI or ${ }^{123 / 131}$ I-MIBG in the localization of SDHxrelated tumors, especially in cases of metastatic disease, where ${ }^{18} \mathrm{~F}$-FDG PET/CT has recently been recommended as one of the first-line functional imaging modalities in this setting [60]. In this sense, from a molecular point of view, recent studies suggest that $\mathrm{SDHx}$ mutations which determine an accumulation of succinate inside PPGL cells, indirectly stimulate glucose uptake, thus explaining the increased ${ }^{18} \mathrm{~F}$-FDG uptake in these tumors, whereas

Tailored Molecular Imaging of

Pheochromocytoma and Paraganglioma in non-SDHx PPGLs, the uptake is usually low to moderate [60]. For instance, an Indian study group recently published the results of a prospective study that included 96 patients, describing the impact of tumor location, biochemical phenotype, malignant nature, and genetics, on sensitivity of ${ }^{18} \mathrm{~F}$-FDG PET/CT and SUVmax values. In fact, they demonstrated that SUVmax of lesions was higher in extra-adrenal locations, in patients with mutations in cluster 1 genes (SDHx and VHL) and in nonepinephrine-secreting rather than those with mutations in cluster 2 genes (RET and NF1) and E-secreting, with significant differences also in terms of sensitivity between clusters with $100 \%$ and $77.7 \%$, respectively [61]. Analogously, Nockel et al. [62] analyzed data from 93 patients with PCC or PGL in order to determine whether routine preoperative ${ }^{18} \mathrm{~F}-\mathrm{FDG} \mathrm{PET} / \mathrm{CT}$ affects surgical management of patients compared to anatomical imaging techniques such as CT and MRI and to examine what clinical and genetic factors may be associated with ${ }^{18} \mathrm{~F}-\mathrm{FDG}$ PET/CT positivity. Their results showed that preoperative ${ }^{18} \mathrm{~F}$ FDG PET/CT identified additional lesions compared to anatomic imaging in 15 cases, eight of which had known germline mutations affecting SDHx, VHL, or FH genes. Therefore, they concluded that incorporating ${ }^{18} \mathrm{~F}-\mathrm{FDG}$ $\mathrm{PET} / \mathrm{CT}$ in the preoperative workup of all patients with PPGLs directly affects surgical management of patients (open approach vs. laparoscopic resection) and should be considered in clinical practice, especially in patients with clinically aggressive and SDHB-associated metastatic tumors [62]. More recently, van Berkel et al. [63] prospectively studied 26 patients with either sporadic or familial PPGL, performing dynamic ${ }^{18} \mathrm{~F}-\mathrm{FDG}$ PET/CT to assess 


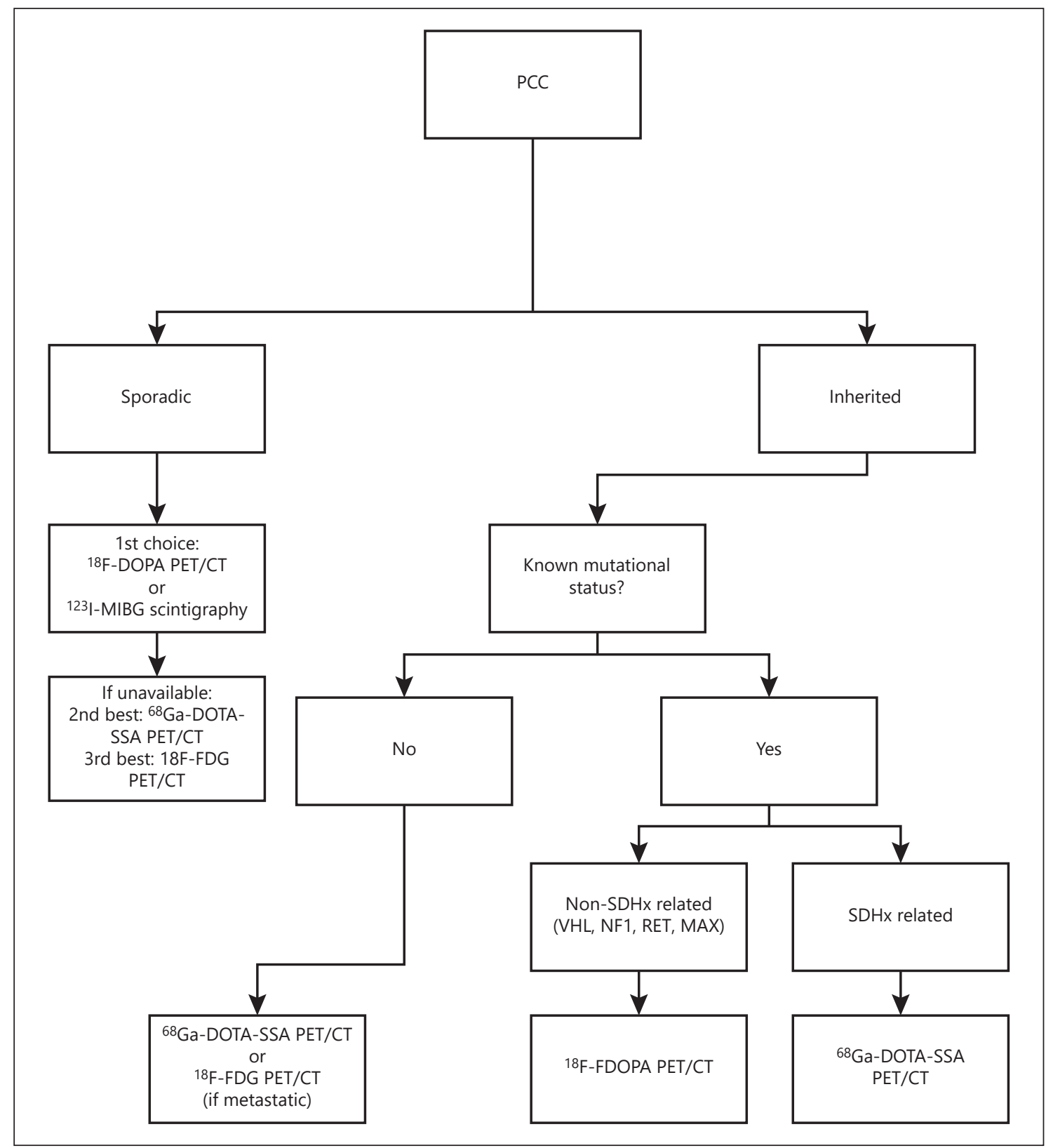

Fig. 3. Proposed flow chart for diagnosis and staging of PCC.

whether ${ }^{18} \mathrm{~F}-\mathrm{FDG}$ pharmacokinetics has added value over static PET to distinguish different genotypes. Thanks to dynamic imaging, they performed quantitative assessment of in vivo glucose metabolic rate which resulted to be higher for hereditary cluster 1 tumors rather than for both cluster 2 and sporadic tumors. Moreover, according to their irreversible 2-tissue compartment model for ${ }^{18} \mathrm{~F}$ FDG metabolism, the glucose phosphorylation rate con- stant (i.e., hexokinase activity) had the same behavior of glucose metabolic rate and SUVmax, being higher in cluster 1 and lower in cluster 2 and sporadic tumors, thus leading them to conclude that dynamic PET/CT provides better in vivo metabolic tumor profiling and allows reliable identification of cluster 1 PPGLs via high ${ }^{18} \mathrm{~F}-\mathrm{FDG}$ phosphorylation rate [63]. 


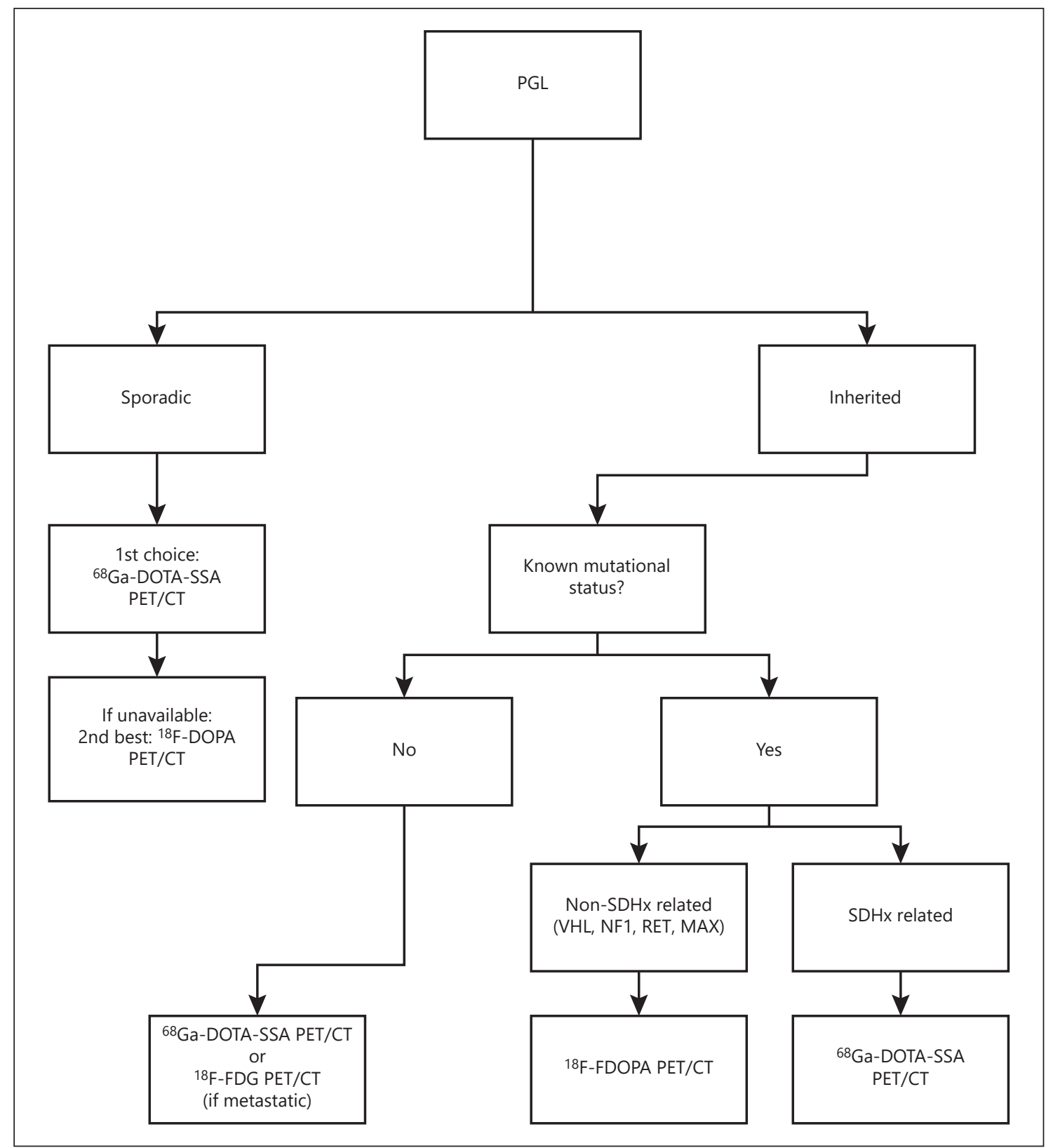

Fig. 4. Proposed flow chart for diagnosis and staging of PGL.

\section{Flow Charts for Diagnosis and Staging of PCC and PGL and Conclusions}

According to current literature data, and in keeping with 2019 EANM/SNMMI Practice Guidelines for radionuclide imaging of PPGLs, we recommend the following flow charts for use in the selection of tracers (Table 3,4) for different clinical scenarios, based on sporadic/inher- ited tumor and known/unknown mutation status for both PCC (shown in Fig. 3) and PGL (shown in Fig. 4). The identification of genetic status allows functional imaging modalities to be personalized, even though both ${ }^{18} \mathrm{~F}$-FDOPA PET/CT and ${ }^{68} \mathrm{Ga}$-DOTA-SSA PET/CT provide excellent results in terms of sensitivity and specificity for primary diagnosis compared to conventional anatomical imaging techniques. Sporadic PCCs are better 
imaged with ${ }^{18} \mathrm{~F}$-FDOPA PET/CT or, if unavailable, with ${ }^{123}$ I-MIBG scintigraphy. It should be noted that the latter is less sensitive and presents more practical constraints; conversely, in case of sporadic PGLs, the best imaging modality is ${ }^{68} \mathrm{Ga}$-DOTA-SSA PET/CT. This is capable of detecting with high specificity even very small lesions that may be overlooked by other imaging modalities, especially HNPGL or extra-adrenal lesions. In terms of mutational status, the most important distinction has to be done for SDHx: non-SDHx tumors are better visualized with ${ }^{68} \mathrm{Ga}$-DOTA-SSA PET/CT rather than ${ }^{18} \mathrm{~F}$-FDOPA PET/ CT, whereas SDHx-related lesions could benefit also of ${ }^{18} \mathrm{~F}-\mathrm{FDG} \mathrm{PET} / \mathrm{CT}$ as an alternative choice (especially when there are suspected metastases), although ${ }^{68} \mathrm{Ga}$ DOTA-SSA PET/CT remains the most sensitive approach. In particular, high ${ }^{18} \mathrm{~F}-\mathrm{FDG}$ phosphorylation rate evaluated via dynamic PET/CT could represent an interesting opportunity for reliable identification of cluster 1 PPGLs as a surrogate of metabolic tumor profiling in cases where biopsy is not feasible.

\section{Acknowledgments}

The authors would like to thank William Russell-Edu, Librarian at the European Institute of Oncology, in Milan, who has linguistically reviewed the manuscript.

\section{Conflict of Interest Statement}

The authors have no conflicts of interest to declare.

\section{Funding Sources}

This research received no funding.

\section{Author Contributions}

Conceptualization: G.L.C; writing - original draft preparation: G.D.D.S.; writing - review and editing: C.M.G. and V.C.; supervision: C.M.G. All the authors have read and agreed to the published version of the manuscript.

\section{References}

1 Neumann HPH, Young WF Jr, Eng C. Pheochromocytoma and paraganglioma. $\mathrm{N}$ Engl J Med. 2019 Aug 8;381(6):552-65.

2 Lam AK. Update on adrenal tumours in 2017 World Health Organization (WHO) of endocrine tumours. Endocr Pathol. 2017;28(3): $213-27$.

3 Lenders JWM, Kerstens MN, Amar L, Prejbisz A, Robledo M, Taieb D, et al. Genetics, diagnosis, management and future directions of research of phaeochromocytoma and paraganglioma: a position statement and consensus of the Working Group on Endocrine Hypertension of the European Society of Hypertension. J Hypertens. 2020;38(8):1443-56.

4 Crona J, Lamarca A, Ghosal S, Welin S, Skogseid B, Pacak K. Genotype-phenotype correlations in pheochromocytoma and paraganglioma: a systematic review and individual patient meta-analysis. Endocr Relat Cancer. 2019 May;26(5):539-50.

5 Nolting S, Bechmann N, Taieb D, Beuschlein F, Fassnacht M, Kroiss M, et al. Personalized management of pheochromocytoma and paraganglioma. Endocr Rev. 2021 Jun 19. Epub ahead of print.

6 Cuccurullo V, Mansi L. Toward tailored medicine (and beyond): the phaeochromocytoma and paraganglioma model. Eur J Nucl Med Mol Imaging. 2012 Aug;39(8):1262-5.

7 Mansi L, Cuccurullo V. Diagnostic imaging in neuroendocrine tumors. J Nucl Med. 2014 Oct;55(10):1576-7.

8 Taieb D, Hicks RJ, Hindie E, Guillet BA, Avram A, Ghedini P, et al. European Associa- tion of Nuclear Medicine practice guideline/ Society of Nuclear Medicine and Molecular Imaging Procedure Standard 2019 for radionuclide imaging of phaeochromocytoma and paraganglioma. Eur J Nucl Med Mol Imaging. 2019 Sep;46(10):2112-37.

9 Jochmanova I, Pacak K. Genomic landscape of pheochromocytoma and paraganglioma. Trends Cancer. 2018;4(1):6-9.

10 Burnichon N, Vescovo L, Amar L, Libé R, de Reynies A, Venisse A, et al. Integrative genomic analysis reveals somatic mutations in pheochromocytoma and paraganglioma. Hum Mol Genet. 2011;20(20):3974-85.

11 Fishbein L, Leshchiner I, Walter V, Danilova L, Robertson AG, Johnson AR, et al. Comprehensive molecular characterization of pheochromocytoma and paraganglioma. Cancer Cell. 2017 Feb 13;31(2):181-93.

12 Fishbein L, Khare S, Wubbenhorst B, DeSloover D, D'Andrea K, Merrill S, et al. Wholeexome sequencing identifies somatic ATRX mutations in pheochromocytomas and paragangliomas. Nat Commun. 2015;6:6140.

13 Flores SK, Estrada-Zuniga CM, Thallapureddy K, Armaiz-Peña G, Dahia PLM. Insights into mechanisms of pheochromocytomas and paragangliomas driven by known or new genetic drivers. Cancers. 2021 Sep 14;13(18): 4602 .

14 Lin PC, Yeh YM, Hsu HP, Chan RH, Lin BW, Chen PC, et al. Comprehensively exploring the mutational landscape and patterns of genomic evolution in hypermutated cancers. Cancers. 2021 Aug 26;13(17):4317.
15 Ma X, Li M, Tong A, Wang F, Cui Y, Zhang $\mathrm{X}$, et al. Genetic and clinical profiles of pheochromocytoma and paraganglioma: a single center study. Front Endocrinol. 2020 Dec 11; 11:574662

16 Gimenez-Roqueplo AP, Dahia PL, Robledo $\mathrm{M}$. An update on the genetics of paraganglioma, pheochromocytoma, and associated hereditary syndromes. Horm Metab Res. 2012 May;44(5):328-33.

17 Crona J, Taïeb D, Pacak K. New perspectives on pheochromocytoma and paraganglioma: toward a molecular classification. Endocr Rev. 2017;38(6):489-515.

18 Geroula A, Deutschbein T, Langton K, Masjkur J, Pamporaki C, Peitzsch M, et al. Pheochromocytoma and paraganglioma: clinical feature-based disease probability in relation to catecholamine biochemistry and reason for disease suspicion. Eur J Endocrinol. 2019; 181(4):409-20.

19 Goldstein RE, O’Neill JA Jr, Holcomb GW 3rd, Morgan WM 3rd, Neblett WW 3rd, Oates JA, et al. Clinical experience over 48 years with pheochromocytoma. Ann Surg. 1999;229(6):755-64.

20 Aygun N, Uludag M. Pheochromocytoma and paraganglioma: from clinical findings to diagnosis. Sisli Etfal Hastan Tip Bul. 2020 Sep 8:54(3):271-80.

21 Cerqueira A, Seco T, Costa A, Tavares M, Cotter J. Pheochromocytoma and paraganglioma: a review of diagnosis, management and treatment of rare causes of hypertension. Cureus. 2020 May 5;12(5):e7969. 
22 Canu L, Parenti G, De Filpo G, Mannelli M. Pheochromocytomas and paragangliomas as causes of endocrine hypertension. Front Endocrinol. 2019 Jun 4;10:333.

23 Chen H, Sippel RS, O'Dorisio MS, Vinik AI, Lloyd RV, Pacak K, et al. The North American Neuroendocrine Tumor Society consensus guideline for the diagnosis and management of neuroendocrine tumors: pheochromocytoma, paraganglioma, and medullary thyroid cancer. Pancreas. 2010 Aug;39(6):775-83.

24 Pacak K. Preoperative management of the pheochromocytoma patient. J Clin Endocrinol Metab. 2007;92(11):4069-79.

25 Yalniz C, Morani AC, Waguespack SG, Elsayes KM. Imaging of adrenal-related endocrine disorders. Radiol Clin North Am. 2020 Nov;58(6):1099-113.

26 Allen BC, Francis IR. Adrenal imaging and intervention. Radiol Clin North Am. 2015 Sep; 53(5):1021-35.

27 Elsayes KM, Elmohr MM, Javadi S, Menias CO, Remer EM, Morani AC, et al. Mimics, pitfalls, and misdiagnoses of adrenal masses on CT and MRI. Abdom Radiol. 2020 Apr; 45(4):982-1000.

28 Elsayes KM, Emad-Eldin S, Morani AC, Jensen CT. Practical approach to adrenal imaging. Urol Clin North Am. 2018 Aug;45(3): 365-87.

29 Taieb D, Timmers HJ, Shulkin BL, Pacak K. Renaissance of 18F-FDG positron emission tomography in the imaging of pheochromocytoma/paraganglioma. J Clin Endocrinol Metab. 2014 Jul;99(7):2337-9.

30 Cuccurullo V, Di Stasio GD, Mansi L. Physiopathological premises to nuclear medicine imaging of pancreatic neuroendocrine tumours. Curr Radiopharm. 2019;12(2):98106.

31 Taïeb D, Jha A, Treglia G, Pacak K. Molecular imaging and radionuclide therapy of pheochromocytoma and paraganglioma in the era of genomic characterization of disease subgroups. Endocr Relat Cancer. 2019;26(11): R627-52.

32 Haddad T, Fard-Esfahani A, Vali R. A review of pediatric neuroendocrine tumors, their detection, and treatment by radioisotopes. Nucl Med Commun. 2021 Jan;42(1):21-31.

33 Pauwels E, Van Aerde M, Bormans G, Deroose CM. Molecular imaging of norepinephrine transporter-expressing tumors: current status and future prospects. Q J Nucl Med Mol Imaging. 2020 Sep;64(3):234-49.

34 Desai H, Borges-Neto S, Wong TZ. Molecular imaging and therapy for neuroendocrine tumors. Curr Treat Options Oncol. 2019 Aug 29;20(10):78.

35 Rozovsky K, Koplewitz BZ, Krausz Y, RevelVilk S, Weintraub M, Chisin R, et al. Added value of SPECT/CT for correlation of MIBG scintigraphy and diagnostic CT in neuroblastoma and pheochromocytoma. AJR Am J Roentgenol. 2008 Apr;190(4):1085-90.
36 Buitenwerf E, Berends AMA, van Asselt ADI, Korteweg T, Greuter MJW, Veeger NJM, et al. Diagnostic accuracy of computed tomography to exclude pheochromocytoma: a systematic review, meta-analysis, and cost analysis. Mayo Clin Proc. 2019;94(10):2040-52.

37 Timmers HJ, Chen CC, Carrasquillo JA, Whatley M, Ling A, Havekes B, et al. Comparison of 18F-fluoro-L-DOPA, 18F-fluorodeoxyglucose, and 18F-fluorodopamine PET and 123I-MIBG scintigraphy in the localization of pheochromocytoma and paraganglioma. J Clin Endocrinol Metab. 2009 Dec; 94(12):4757-67.

38 Jha A, Ling A, Millo C, Gupta G, Viana B, Lin FI, et al. Superiority of $68 \mathrm{Ga}$-DOTATATE over 18F-FDG and anatomic imaging in the detection of succinate dehydrogenase mutation (SDHx)-related pheochromocytoma and paraganglioma in the pediatric population. Eur J Nucl Med Mol Imaging. 2018;45(5): 787-97.

39 Timmers HJ, Hadi M, Carrasquillo JA, Chen CC, Martiniova L, Whatley M, et al. The effects of carbidopa on uptake of 6-18F-FluoroL-DOPA in PET of pheochromocytoma and extraadrenal abdominal paraganglioma. J Nucl Med. 2007 Oct;48(10):1599-606.

40 Fottner C, Helisch A, Anlauf M, Rossmann H, Musholt TJ, Kreft A, et al. 6-18F-fluoro-L-dihydroxyphenylalanine positron emission tomography is superior to 123I-metaiodobenzyl-guanidine scintigraphy in the detection of extraadrenal and hereditary pheochromocytomas and paragangliomas: correlation with vesicular monoamine transporter expression. J Clin Endocrinol Metab. 2010 Jun;95(6): 2800-10.

41 Noordzij W, Glaudemans A, Schaafsma M, van der Horst-Schrivers ANA, Slart R, van Beek AP, et al. Adrenal tracer uptake by $18 \mathrm{~F}$ FDOPA PET/CT in patients with pheochromocytoma and controls. Eur J Nucl Med Mol Imaging. 2019 Jul;46(7):1560-6.

42 Gabriel S, Blanchet EM, Sebag F, Chen CC, Fakhry N, Deveze A, et al. Functional characterization of nonmetastatic paraganglioma and pheochromocytoma by $18 \mathrm{~F}-\mathrm{FDOPA}$ PET: focus on missed lesions. Clin Endocrinol. 2013 Aug;79(2):170-7.

43 Reubi JC. Peptide receptors as molecular targets for cancer diagnosis and therapy. Endocr Rev. 2003;24:389-427.

44 Barnett P. Somatostatin and somatostatin receptor physiology. Endocrine. 2003;20(3): 255-64.

45 Reubi JC, Waser B, Schaer JC, Laissue JA. Somatostatin receptor sst1-sst5 expression in normal and neoplastic human tissues using receptor autoradiography with subtype-selective ligands. Eur J Nucl Med. 2001;28(7):83646.

46 Yordanova A, Biersack HJ, Ahmadzadehfar $\mathrm{H}$. Advances in molecular imaging and radionuclide therapy of neuroendocrine tumors. J Clin Med. 2020 Nov 16;9(11):3679.
47 Mundschenk J, Unger N, Schulz S, Hollt V, Schulz S, Steinke R, et al. Somatostatin receptor subtypes in human pheochromocytoma: subcellular expression pattern and functional relevance for octreotide scintigraphy. J Clin Endocrinol Metab. 2003 Nov;88(11):5150-7.

48 Han S, Suh CH, Woo S, Kim YJ, Lee JJ. Performance of $68 \mathrm{Ga}-\mathrm{DOTA}$-conjugated somatostatin receptor-targeting peptide PET in detection of pheochromocytoma and paraganglioma: a systematic review and metaanalysis. J Nucl Med. 2019 Mar;60(3):369-76.

49 Kwekkeboom DJ, Kam BL, van Essen M, Teunissen JJ, van Eijck CH, Valkema R, et al. Somatostatin-receptor-based imaging and therapy of gastroenteropancreatic neuroendocrine tumors. Endocr Relat Cancer. 2010; 17(1):R53-73.

50 Cuccurullo V, Di Stasio GD, Prisco MR, Mansi L. Is there a clinical usefulness for radiolabeled somatostatin analogues beyond the consolidated role in NETs? Indian J Radiol Imaging. 2017 Oct-Dec;27(4):509-16.

51 Ambrosini V, Campana D, Polverari G, Peterle C, Diodato S, Ricci C, et al. Prognostic value of 68Ga-DOTANOC PET/CT SUVmax in patients with neuroendocrine tumors of the pancreas. J Nucl Med. 2015 Dec;56(12): 1843-8.

$52 \mathrm{Ma} \mathrm{H}, \mathrm{Kan}$ Y, Yang JG. Clinical value of 68GaDOTA-SSTR PET/CT in the diagnosis and detection of neuroendocrine tumors of unknown primary origin: a systematic review and meta-analysis. Acta Radiol. 2021 Sep; 62(9):1217-28.

53 Ambrosini V, Nanni C, Fanti S. The use of gallium-68 labeled somatostatin receptors in PET/CT imaging. PET Clin. 2014;9(3):323-9.

54 Maurice JB, Troke R, Win Z, Ramachandran R, Al-Nahhas A, Naji M, et al. A comparison of the performance of 68Ga-DOTATATE PET/CT and 123I-MIBG SPECT in the diagnosis and follow-up of phaeochromocytoma and paraganglioma. Eur J Nucl Med Mol Imaging. 2012 Aug;39(8):1266-70.

55 Janssen I, Chen CC, Taieb D, Patronas NJ, Millo CM, Adams KT, et al. 68Ga-DOTATATE PET/CT in the localization of head and neck paragangliomas compared with other functional imaging modalities and CT/ MRI. J Nucl Med. 2016 Feb;57(2):186-91.

56 Janssen I, Blanchet EM, Adams K, Chen CC, Millo CM, Herscovitch P, et al. Superiority of [68Ga]-DOTATATE PET/CT to other functional imaging modalities in the localization of SDHB-associated metastatic pheochromocytoma and paraganglioma. Clin Cancer Res. 2015 Sep 1;21(17):3888-95.

57 Archier A, Varoquaux A, Garrigue P, Montava M, Guerin C, Gabriel S, et al. Prospective comparison of $68 \mathrm{Ga}-\mathrm{DOTATATE}$ and $18 \mathrm{~F}$ FDOPA PET/CT in patients with various pheochromocytomas and paragangliomas with emphasis on sporadic cases. Eur J Nucl Med Mol Imaging. 2016;43(7):1248-57.
Tailored Molecular Imaging of Pheochromocytoma and Paraganglioma
Neuroendocrinology 2022;112:927-940 DOI: $10.1159 / 000522089$ 
58 Cuccurullo V, Prisco MR, Di Stasio GD, Mansi L. Nuclear medicine in patients with NET: radiolabeled somatostatin analogues and their brothers. Curr Radiopharm. 2017;10(2): 74-84.

59 Baum RP, Kulkarni HR, Carreras C. Peptides and receptors in image-guided therapy: theranostics for neuroendocrine neoplasms. Semin Nucl Med. 2012;42(3):190-207.
60 Fikri AS, Kroiss A, Ahmad AZ, Zanariah H, Lau WF, Uprimny C, et al. Localization and prediction of malignant potential in recurrent pheochromocytoma/paraganglioma (PCC) PGL) using 18F-FDG PET/CT. Acta Radiol. 2014 Jun;55(5):631-40.

61 Tiwari A, Shah N, Sarathi V, Malhotra G, Bakshi G, Prakash G, et al. Genetic status determines 18F-FDG uptake in pheochromocyto$\mathrm{ma}$ /paraganglioma. J Med Imaging Radiat Oncol. 2017 Dec;61(6):745-52.
62 Nockel P, El Lakis M, Gaitanidis A, Merkel R, Patel D, Nilubol N, et al. Preoperative 18FFDG PET/CT in pheochromocytomas and paragangliomas allows for precision surgery. Ann Surg. 2019 Apr;269(4):741-7.

63 van Berkel A, Vriens D, Visser EP, Janssen MJR, Gotthardt M, Hermus A, et al. Metabolic subtyping of pheochromocytoma and paraganglioma by 18 F-FDG pharmacokinetics using dynamic PET/CT scanning. J Nucl Med. 2019 Jun;60(6):745-51. 